Research Article

\title{
Effects of Noninvasive Low-Intensity Focus Ultrasound Neuromodulation on Spinal Cord Neurocircuits In Vivo
}

\author{
Ye-Hui Liao $\mathbb{D},{ }^{1,2}$ Mo-Xian Chen $\left(\mathbb{D},{ }^{1}\right.$ Shao-Chun Chen, ${ }^{1}$ Kai-Xuan Luo, ${ }^{1}$ Bin Wang $\mathbb{D}^{1}{ }^{1}$ \\ Yao Liu (D), ${ }^{1}$ and Li-Juan Ao (iD ${ }^{1}$ \\ ${ }^{1}$ School of Rehabilitation, Kunming Medical University, Kunming 650500, Yunnan Province, China \\ ${ }^{2}$ Department of Orthopaedics, Affiliated Hospital of Southwest Medical University, Luzhou 646000, Sichuan Province, China \\ Correspondence should be addressed to Yao Liu; 175381219@qq.com and Li-Juan Ao; aolijuan@kmmu.edu.cn
}

Received 7 September 2021; Revised 8 October 2021; Accepted 26 October 2021; Published 27 November 2021

Academic Editor: Feng Zhang

Copyright (C) 2021 Ye-Hui Liao et al. This is an open access article distributed under the Creative Commons Attribution License, which permits unrestricted use, distribution, and reproduction in any medium, provided the original work is properly cited.

\begin{abstract}
Although neurocircuits can be activated by focused ultrasound stimulation, it is unclear whether this is also true for spinal cord neurocircuits. In this study, we used low-intensity focused ultrasound (LIFU) to stimulate lumbar 4-lumbar 5 (L4-L5) segments of the spinal cord of normal Sprague Dawley rats with a clapper. The activation of the spinal cord neurocircuits enhanced soleus muscle contraction as measured by electromyography (EMG). Neuronal activation and injury were assessed by EMG, western blotting (WB), immunofluorescence, hematoxylin and eosin (H\&E) staining, Nissl staining, enzyme-linked immunosorbent assay (ELISA), immunohistochemistry (IHC), somatosensory evoked potentials (SEPs), motor evoked potentials (MEPs), and the Basso-Beattie-Bresnahan locomotor rating scale. When the LIFU intensity was more than $0.5 \mathrm{MPa}$, LIFU stimulation induced soleus muscle contraction and increased the EMG amplitudes $(P<0.05)$ and the number of c-fos- and GAD65-positive cells $(P<0.05)$. When the LIFU intensity was $3.0 \mathrm{MPa}$, the LIFU stimulation led to spinal cord damage and decreased SEP amplitudes for electrophysiological assessment $(P<0.05)$; this resulted in coagulation necrosis, structural destruction, neuronal loss in the dorsal horn by H\&E and Nissl staining, and increased expression of GFAP, IL- $1 \beta$, TNF- $\alpha$, and caspase- 3 by IHC, ELISA, and WB $(P<0.05)$. These results show that LIFU can activate spinal cord neurocircuits and that LIFU stimulation with an irradiation intensity $\leq 1.5 \mathrm{MPa}$ is a safe neurostimulation method for the spinal cord.
\end{abstract}

\section{Introduction}

Neurostimulation technology, including focused ultrasound stimulation, transcranial magnetic stimulation (TMS), deep brain stimulation (DBS), and optogenetic stimulation, has become an important neuromodulation method for various neurological conditions. TMS is a noninvasive or minimally invasive neurostimulation technique, which has been widely used for neuromodulation. TMS has advantages for superficial brain regions, while its effects on deeper brain regions are limited given its poor spatial resolution [1]. DBS, including electrical stimulation or epidural electrical stimulation, is a commonly used neurostimulation method. Epidural stimulation of the spinal cord plays a positive role in the functional recovery of the injured spinal cord $[2,3]$. However, because of the highly diffuse electric field and lower spatial resolution, it is difficult to locate a specific area of interest using electrical stimulation [4]. Moreover, surgery is also required, and the electrode is inserted in or on the cerebral cortex or epidural space for electrical stimulation [2]. Optogenetic stimulation also requires invasive procedures and genetic manipulation, which are not feasible in humans $[5,6]$. Recently, focused ultrasound has attracted much attention and interest due to its high spatial resolution, noninvasive neurostimulation, and effective stimulation of the deep tissues with submillimeter static resolution $[7,8]$. Consequently, it has become an alternative modality for neuromodulation [9].

Ultrasound is a mechanical pressure wave with a frequency of $>20 \mathrm{kHz}$, which can be transmitted through bone and soft tissues. An acoustic intensity $<500 \mathrm{mw} / \mathrm{cm}^{2}$ (lowintensity ultrasound) has shown significant biological effects 
without producing thermal effects or tissue damage [10, 11]. Moreover, many studies have confirmed that low-intensity ultrasound stimulation can inhibit or stimulate neurons both in vitro and in vivo. Recent research has shown that low-intensity, low-frequency ultrasound stimulation of hippocampal slices excites the neurons and network activity by activating voltage-gated sodium and calcium channels $[12,13]$. Further studies have confirmed that low-intensity focused ultrasound (LIFU) activates neurocircuits in both model organisms and humans. Indeed, pulsed focused ultrasonic stimulation has been shown to effectively induce nerve responses and action potentials in the giant fibers of invertebrate animals $[14,15]$. Moreover, precise stimulation of the deep brain nuclei and modulation of brain neuronal activity [16-19] have been shown to induce muscle contraction of the limbs of rats and rabbits, as verified by electromyography (EMG) $[20,21]$. Primate studies have also found that focused ultrasound stimulation of the brains of two awake macaque rhesus monkeys significantly modulates high-level cognitive behavior [22]. Gavrilov [23] confirmed that ultrasound could induce tactile, thermal, and pain sensations by activation of somatosensory neurons in humans. As a result, low-intensity ultrasound, especially LIFU, has gained widespread attention as a potential clinical neuromodulation technology.

The spinal cord contains complex neurocircuits, the stimulation of which is an important method to treat chronic spine-related conditions, such as failed back surgery syndrome, complex regional pain syndrome, painful diabetic neuropathy, and spinal cord injury [3, 24-28]. Recently, there has been growing interest in ultrasound neuromodulation; however, little is known about whether spinal cord stimulation with LIFU can activate or inhibit spinal cord neurocircuits and whether such stimulation causes injury to the spinal cord. Activation of the spinal cord neurocircuits can induce muscle contractions and produce action potentials, which can be measured by EMG [20]. In this study, LIFU was used to stimulate the L4-L5 segments of the spinal cord in Sprague Dawley (SD) rats, and EMG was used to measure the stimulation success of the spinal cord neurocircuits. Hematoxylin and eosin (H\&E) staining, Nissl staining, and biomarker tests were used to evaluate the safety of LIFU stimulation.

\section{Materials and Methods}

2.1. Animals. Adult male SD rats weighing 220-300 g were acquired from the Kunming Laboratory Animal Center for experimental use. All of the animal protocols were approved by the Animal Ethics Committee of Kunming Medical University (KMMU2020352). All rats were housed at a temperature of $25 \pm 2^{\circ} \mathrm{C}$ with a 12/12-h light/dark cycle, and all rats had free access to food and water.

2.2. Experimental Protocol. After 1 week of adaptation, the SD rats were used for the experiment. The study comprised two protocols. The first study (Study I, $n=6$ ) was designed to test the activation of the spinal cord neurocircuits by LIFU stimulation. In this study, the rats were anesthetized with isoflurane $(1.5 \%)$, and EMG was used to measure the recruitment of the soleus (Sol) muscle when the rats received different acoustic pressure stimulations (radiation intensity $(\mathrm{RI})=0 \mathrm{MPa} \quad\left(I_{\text {spta }}=0 \mathrm{mw} / \mathrm{cm}^{2}\right), \quad 0.5 \mathrm{MPa} \quad\left(I_{\text {spta }}=60 \mathrm{mw} /\right.$ $\left.\mathrm{cm}^{2}\right), \quad 1.0 \mathrm{MPa} \quad\left(I_{\text {spta }}=180 \mathrm{~mW} / \mathrm{cm}^{2}\right), \quad 1.5 \mathrm{MPa}$ $\left(I_{\text {spta }}=320 \mathrm{mw} / \mathrm{cm}^{2}\right), 2.0 \mathrm{MPa}\left(I_{\text {spta }}=400 \mathrm{mw} / \mathrm{cm}^{2}\right), 2.5 \mathrm{MPa}$ $\left(I_{\text {spta }}=500 \mathrm{mw} / \mathrm{cm}^{2}\right)$, or $\left.3.0 \mathrm{MPa}\left(I_{\text {spta }}=600 \mathrm{mw} / \mathrm{cm}^{2}\right)\right)$ with $20 \%$ duty cycle (DC). There was a 5 min interval between different ultrasonic parameter tests. The study protocol is shown in Figure 1(a).

In the second study (Study II, $n=42$ ), the safety of LIFU stimulation was detected by electrophysiology, neuromotor function, H\&E staining, Nissl staining, enzyme-linked immunosorbent assay (ELISA), and biomarker tests. The rats were divided into four groups as follows: the negative LIFU stimulation group (LIFU ${ }^{-}$group, $\mathrm{RI}=0 \mathrm{MPa}, n=6$ ), the LIFU stimulation 1 group $\left(\mathrm{LIFU}^{+1}\right.$ group, $\mathrm{RI}=0.5 \mathrm{MPa}$, $n=12$ ), the LIFU stimulation 2 group $\left(\mathrm{LIFU}^{+2}\right.$ group, $\mathrm{RI}=1.5 \mathrm{MPa}, n=12$ ), and the LIFU stimulation 3 group $\left(\mathrm{LIFU}^{+3}\right.$ group, RI $\left.=3.0 \mathrm{MPa}, n=12\right)$. After anesthesia with isoflurane $(1.5 \%)$, the rats were stimulated with LIFU for $20 \mathrm{~min}$. After LIFU treatment, the safety test was performed according to the protocol shown in Figure 1(b).

2.3. LIFU Stimulation. The LIFU stimulation procedure was performed as follows [29]: after anesthesia, the rats were fixed on a table, and the hair on their backs was removed using a depilating cream to expose the L4-L5 segments of the spinal cord. An ultrasound probe was fixed to the back of the L4-L5 spinal cord segment with a clapper. An ultrasound gel was used to fill the space between the skin and the ultrasound probe. A waveform signal was generated by a twochannel function/arbitrary waveform generator (DG4202, RIGOL, China) and was amplified with a $50 \mathrm{~W}$ power amplifier (Dahan Radio Studio, China). The amplified signal activated the ultrasonic probe. Channel I was set to deliver the wave signal with cycles of $1000 \mathrm{~Hz} / \mathrm{s}$, a burst duration of $1 \mathrm{~s}$, and a $20 \%$ total duty cycle. Channel II was set to deliver the ultrasound probe with a frequency of $4 \mathrm{MHz}$ and 800 cycles for every pulse period (Figures 2(a)-2(b)). The acoustic pressure of the ultrasound was measured using a hydrophone (Onda HNP-1000, ONDA Corporation, Sunnyvale, CA, USA) in a water tank (Figures 2(c)-2(e)).

\subsection{Neuromotor Function Assessment. The Bas-} so-Beattie-Bresnahan locomotor rating scale (BBB scale) was used for the hind limb neuromotor function assessment pre- and post-LIFU. The assessment was performed as previously described [30]. The BBB rating scale assesses the coordination of limb movement, paw placement, and tail balance. The scale ranges from 0 to 21 points, where 0 is defined as no visible movement of the legs, and 21 is defined as normal neuromotor function, i.e., the rat can walk continually on the paws, with consistent plantar stepping, coordinated gait, trunk stability, a cocked tail, and parallel throughout the stance. 


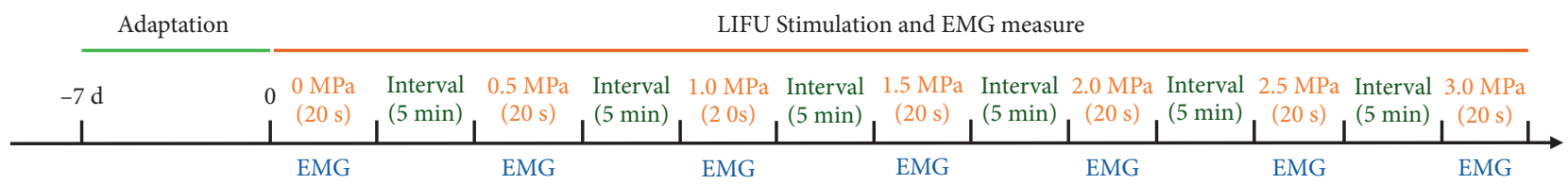

(a)

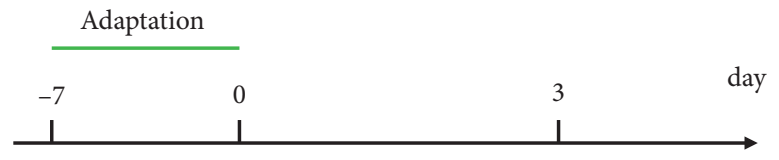

LIFU Stimulation

(1) SEPs/MEPs;

(2) IF: c-Fos, GAD65;

(3) $\mathrm{H} \& \mathrm{E}$;

(1) BBB score;

(4) WB: Caspase3, Bcl-2;

(5) ELISA: IL- $1 \beta$, TNF- $\alpha$;

(2) Nissl;

(3) IHC : GFAP;

(4) WB: Caspase3, Bcl-2;

Radiation Intensity Level:

LIFU ${ }^{-}$group: $\mathrm{RI}=0 \mathrm{Mpa}, \mathrm{n}=6$;

LIFU ${ }^{1+}$ group: $\mathrm{RI}=0.5 \mathrm{Mpa}, \mathrm{n}=12$;

LIFU ${ }^{2+}$ group: $\mathrm{RI}=1.5 \mathrm{Mpa}, \mathrm{n}=12$;

LIFU ${ }^{3+}$ group: $\mathrm{RI}=3.0 \mathrm{Mpa}, \mathrm{n}=12$;

(b)

FIGURE 1: Timeline of study I (a) and II (b) experimental protocols. The rats were killed, and the safety was examined on days 0 and 3 postlow-intensity focused ultrasound (LIFU) stimulation.

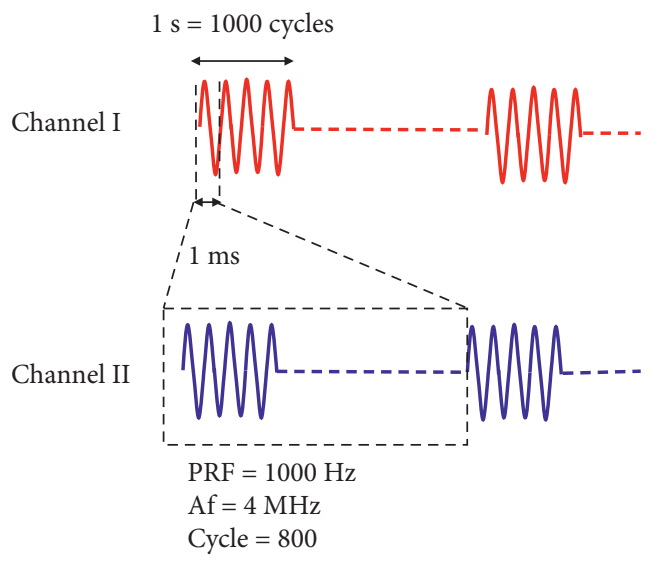

(a)

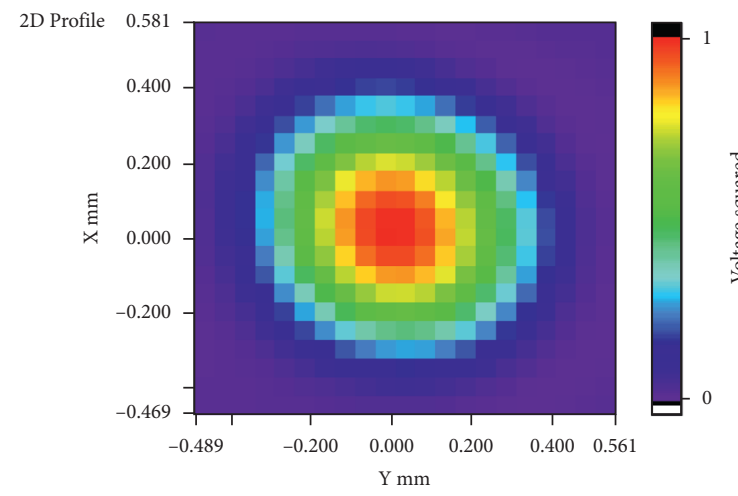

(c)

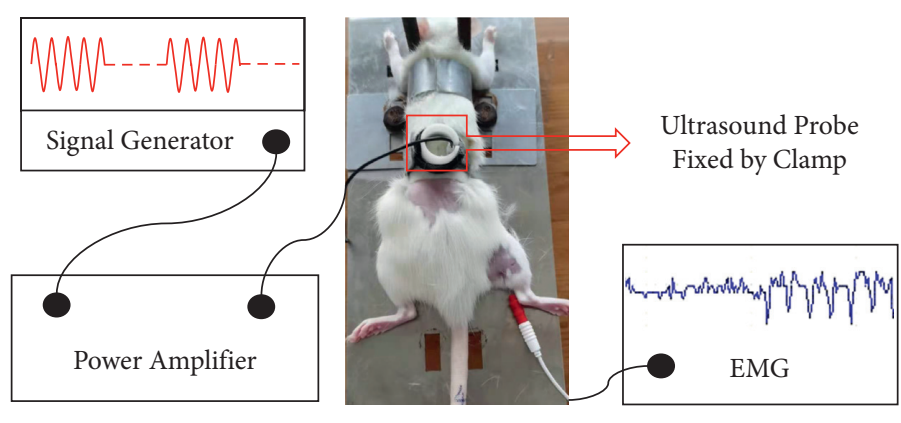

(b)

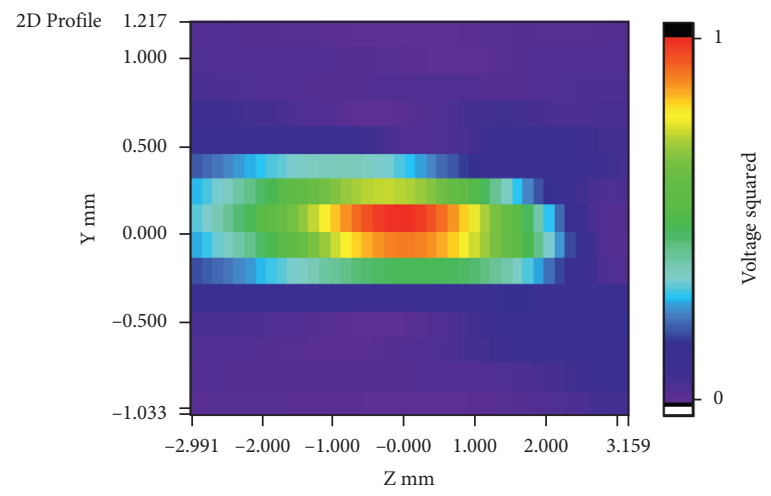

(d)

Figure 2: Continued. 


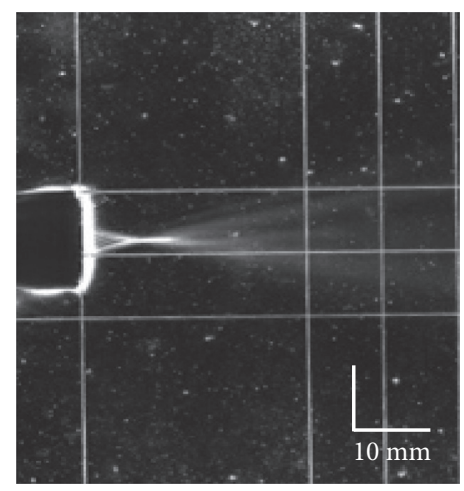

(e)

FIGURE 2: (a) Schematic of the LIFU pulsing strategy. (b) Schematic of LIFU stimulation of the spinal cord and electromyography (EMG) examination. The signal was generated by the generator, amplified by the amplifier, and then converted into an acoustic signal by the ultrasound probe. The ultrasound probe was fixed by the clamp on the back of the rats at the segment L4-L5 spinal cord level. The recruitment of the soleus muscle was recorded at the time of LIFU spinal cord stimulation. (c-e) Parameters of focal ultrasound, including the acoustic-intensity distribution map from transverse and sagittal planes.

2.5. Electrophysiology Test. The recruitment of Sol muscle was used to assess the activation of spinal neurocircuits and was measured by EMG using an electromyographic evoked potentiometer (Neuropack ${ }^{\circledR}$ S1 MEB-9400, Nihon Kohden, Japan). EMG was conducted as described previously [31, 32]. Briefly, the EMG signal was measured by a concentric circular electrode that was percutaneously inserted into the Sol, and the reference electrode was percutaneously inserted into the tail. The EMG signal was recorded at the same time as the LIFU stimulation of the spinal cord and was filtered at $200 \mathrm{~Hz}-5 \mathrm{kHz}$ (Figure 2(b)). The amplitude (intensity of recruitment of Sol) was measured between consecutive peaks, i.e., from the positive peak to the neighboring negative peak $(\mu \mathrm{V})$.

Somatosensory evoked potentials (SEPs) and motor evoked potentials (MEPs) were used to assess the conduction function after the white and gray matter injury of the spinal cord [33]. For the SEP test [34, 35], the recording electrode was placed on the left sensorimotor cortex, the reference electrode was inserted under the skin of the nose, the stimulation electrodes were inserted under the skin of the right ankle, and the ground needle electrode was percutaneously placed in the tail. The stimulation involved sine impulses with a trigger frequency of $2 \mathrm{~Hz}$ and an intensity of $3 \mathrm{~mA}$. Signal data (including the latency and amplitude) were recorded from the electrode placed on the sensorimotor cortex and were filtered with a bandpass $(10-2000 \mathrm{~Hz})$; we recorded 100 evoked potentials on average for two times with $5 \mathrm{~min}$ interval. The latency was defined as the time ( $\mathrm{ms}$ ) between the onset of the stimulus artifact and the first peak (positive or negative). The amplitude $(\mu \mathrm{V})$ was measured from the positive peak to the negative peak.

For the MEP test, the stimulation electrode was placed on the left motor cortex, the recording electrode was percutaneously inserted into the right fifth palmar interosseous muscle, and the reference and ground needle electrodes were placed as outlined for the SEP test. The stimulation involved sine impulses with an intensity of
$4 \mathrm{~mA}$. An analog amplifier (Model 1700 Differential AC Amplifier, AM Systems, USA) was used for amplification $(100 \times)$, filtering $(10 \mathrm{~Hz}-10 \mathrm{kHz}$, bandpass), and recording of MEP signals. The latency was defined as the time (ms) between the onset of the stimulus artifact and the first peak (positive or negative).

The EMG, SEPs, and MEPs data were visualized and recorded on a computer for further analysis using a software interface (Signal, Cambridge Electronics Design Ltd., United Kingdom).

2.6. Tissue Preparation. After neuromotor function and electrophysiology assessment, the rats were killed by an overdose of $1 \%$ sodium pentobarbital ( $40 \mathrm{mg} / \mathrm{kg}$ ). Then, the tissues were collected for western blot, ELISA, and H\&E staining, Nissl staining, immunofluorescence staining, and IHC staining. For western blot and ELISA, L4-L5 spinal cord segments were immediately collected and stored at $-80^{\circ} \mathrm{C}$ until use. For the $\mathrm{H} \& \mathrm{E}$ staining, Nissl staining, immunofluorescence staining, and IHC staining, the rats were perfused with $200 \mathrm{~mL}$ of $0.1 \mathrm{M}$ phosphate-buffered saline (PBS) and then with $200 \mathrm{~mL}$ PBS with $4 \%$ paraformaldehyde ( $\mathrm{pH}$ 7.4). The L4-L5 spinal cord segments were collected, fixed in $4 \%$ paraformaldehyde overnight, dehydrated, and embedded in paraffin. Finally, transverse section slices $(5 \mu \mathrm{m}$ thick) of the spinal cord were prepared for $\mathrm{H} \& \mathrm{E}$ staining, Nissl staining, immunofluorescence staining, and IHC staining.

2.7. Western Blotting. The spinal cord tissue $(0.1 \mathrm{~g})$ was dissected, ultrasonically homogenized, and lysed with PIPA buffer (RIPA : PMSF $=1 \mathrm{~mL}: 10 \mu \mathrm{L}$ ) on ice for $30 \mathrm{~min}$. After centrifugation at $12000 \mathrm{r} / \mathrm{min}$ for $20 \mathrm{~min}$ at $4^{\circ} \mathrm{C}$, the supernatant was collected. The concentration of the total protein was quantified using a bicinchoninic acid assay (Enhanced BCA Protein Assay Kit, Beyotime, China), and all samples were equalized to $30 \mu \mathrm{g} / 10 \mu \mathrm{L}$. The samples $(30 \mu \mathrm{g}$ total protein) were resolved by $10 \%$ sodium dodecyl sulfate- 
polyacrylamide gel electrophoresis (SDS-PAGE) and transferred to polyvinylidene difluoride membranes (PVDFs, Millipore, MA, USA). The membranes were blocked with $5 \%$ fat-free milk at room temperature for $2 \mathrm{~h}$ and incubated with primary antibodies at $4^{\circ} \mathrm{C}$ with gentle shaking overnight. The primary antibodies included polyclonal antibodies against caspase-3 $(1: 2000$, Proteintech, USA), polyclonal antibodies against $\mathrm{Bcl}-2(1: 2000$, Proteintech, USA), and $\beta$-actin $(1: 2000$, Santa, USA). Following incubation, the membranes were incubated with the secondary antibody, peroxidase-conjugated AffiniPure goat anti-mouse/rabbit IgG $(\mathrm{H}+\mathrm{L})(1: 2000$, ZSGB-BIO, China) for $2 \mathrm{~h}$ at room temperature. Finally, the protein bands were visualized and quantified using enhanced chemiluminescence (Tanon, Shanghai, China) and the image processing system ImageJ (Rawak Software, Stuttgart, Germany). The protein concentrations were normalized to $\beta$-actin.

2.8. ELISA for IL-1 $\beta$ and TNF- $\alpha$. The expression levels of the inflammatory factors IL- $1 \beta$ and TNF- $\alpha$ in the spinal cord were examined after ultrasound stimulation. Spinal cord tissue $(0.1 \mathrm{~g})$ was cut into pieces with ophthalmic scissors after adding precooled $0.1 \mathrm{M}$ PBS $(1 \mathrm{~mL})$. Then, the cut tissues were placed in a glass homogenizer and homogenized on ice for $8 \mathrm{~min}$. After centrifugation at $4^{\circ} \mathrm{C}$ and $5000 \mathrm{r} / \mathrm{min}$ for $5 \mathrm{~min}$, the supernatant was obtained, and the total protein concentration was quantified by the bicinchoninic acid assay (Enhanced BCA Protein Assay Kit, Beyotime, China). The ELISA was performed in accordance with the instructions for IL-1 $\beta$ (Bioswamp, RA20020, China: http:// www.bio-swamp.com/upload/file/201711/

1510793514933161.pdf) and TNF- $\alpha$ (Bioswamp, RA20035, China: http://www.bio-swamp.com/upload/file/201910/ 1571017094579621.pdf).

2.9. HぬE Staining and Nissl Staining. $H \& E$ staining and Nissl staining were used to assess the safety of LIFU for use in the spinal cord. The H\&E staining was performed as follows: the slices were dewaxed, dehydrated, stained with H\&E solution, cleared with xylene, and mounted with resin. Toluidine blue was used to stain for Nissl using the following procedure: the slices were dewaxed, rinsed with tap water, treated with toluidine blue, dehydrated, and sealed with neutral gum. Images of $\mathrm{H} \& \mathrm{E}$ staining and Nissl staining were captured using an optical microscope (Olympus Corporation, Tokyo, Japan). The Nissl substance was identified as navy or dark blue with a light blue or light background.

\subsection{Immunofluorescence Staining and IHC Staining. For} immunofluorescence staining and IHC staining, paraffin sections ( $5 \mu \mathrm{m}$ thick) were prepared. First, the sections were subjected to dewaxing, antigen repair, and $\mathrm{H}_{2} \mathrm{O}_{2}$ elimination of endogenous peroxidase. For immunofluorescence staining, the slices were incubated with 5\% goat serum and $0.03 \%$
Triton X-100 in $0.1 \mathrm{M}$ PBS for $2 \mathrm{~h}$. Then, the slices were incubated with primary antibodies, including monoclonal antibodies against $c$-fos $(1: 200$, Proteintech, USA) and GAD65 ( $1: 200$, CST, USA), at $4^{\circ} \mathrm{C}$ overnight. Following incubation, the slices were incubated with secondary antibodies, including anti-rabbit IgG $(\mathrm{H}+\mathrm{L}), \mathrm{F}\left(\mathrm{ab}^{\prime}\right) 2$ fragment (Alexa Fluor ${ }^{\circledR} 594$ Conjugate) and anti-mouse $\operatorname{IgG}(\mathrm{H}+\mathrm{L})$, $\mathrm{F}\left(\mathrm{ab}^{\prime}\right) 2$ fragment (Alexa Fluor ${ }^{\circledR} 488$ Conjugate) at room temperature in the dark for $2 \mathrm{~h}$. After $3 \times 10 \mathrm{~min}$ washing with PBS, the sections were incubated with 4', 6-diamidino2-phenylindole (DAPI; Sigma, USA). The images were captured via a fluorescence microscope (Olympus Corporation, Tokyo, Japan), and ImageJ software (NIH, Bethesda, $M D$, USA) was used to quantify the number of positive cells.

For IHC, the slides were incubated with the primary antibody GFAP $\left(1: 1000\right.$, Cell Signaling, USA) at $4^{\circ} \mathrm{C}$ overnight. Then, the slides were incubated with secondary antibody labeled with poly-HRP anti-rabbit IgG $(1: 1$; Beijing Zhongshan Golden Bridge Biotechnology) for $50 \mathrm{~min}$. After staining with DAB for $20 \mathrm{~s}$ and counterstaining with hematoxylin for $8 \mathrm{~min}$ at room temperature, the images were captured via a light microscope (Olympus Corporation, Tokyo, Japan), and ImageJ software (Rawak Software) was used to quantify the density of positive regions.

2.11. Statistical Analyses. The data are presented as the mean \pm standard error of mean (SEM). SPSS 23.0 (IBM Corp., Armonk, NY, USA) was used for all statistical analyses, and GraphPad Prism software version 8.0 (GraphPad Software Inc., San Diego, CA, USA) was used to prepare the graphs. The amplitude of EMG, the gray intensity of western blot, the number of positive cells in immunofluorescence, and the density data for IHC were calculated. After verifying that all data satisfied the normality of distribution, differences among different intensities of stimulation were determined using analysis of variance (ANOVA). When an ANOVA showed a significant difference, Fisher's protected least significant difference (LSD) tests were used for pairwise comparisons. Differences between pre- and post-LIFU stimulation were analyzed using paired $t$-tests. Two-tailed $P$ values $<0.05$ were considered statistically significant.

\section{Results}

3.1. LIFU Stimulation Enhances the Recruitment of Sol Muscle. As shown in Figure 3, LIFU simulation of the spinal cord enhanced the recruitment of Sol muscle. When the LIFU intensity was $>0.5 \mathrm{MPa}$, the recruitment of Sol muscle was measured on EMG. With the increase in the stimulation intensity, the recruitment intensity (Amp, $\mu \mathrm{V})$ of the Sol muscle also increased (Supplementary Figure 1). When the stimulation intensity was $>1.0 \mathrm{MPa}$, LIFU ON induced significant muscle recruitment and the EMG amplitude was significantly higher than that at LIFU OFF stimulation $(P<0.05)$. There was no significant difference in the amplitude among LIFU OFF time point $(P>0.05)$ (Supplementary Figure 1). 


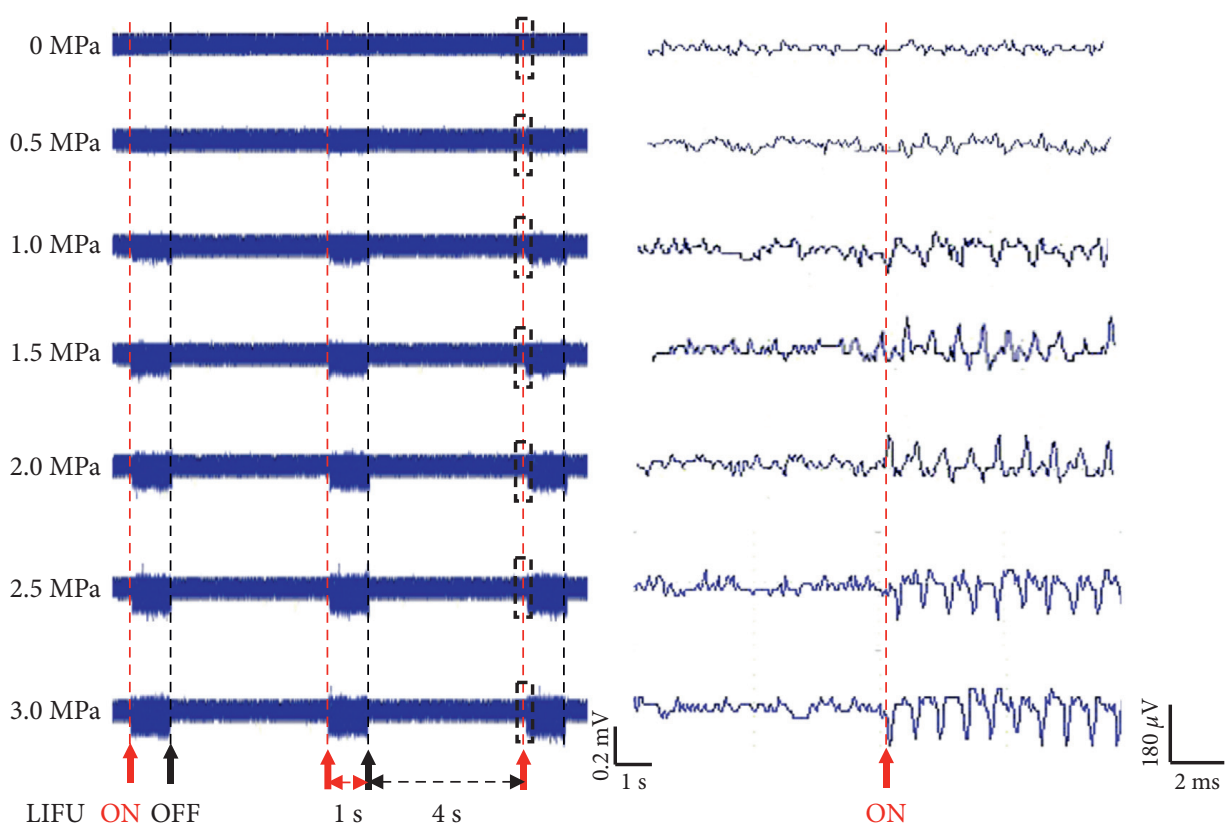

(a)

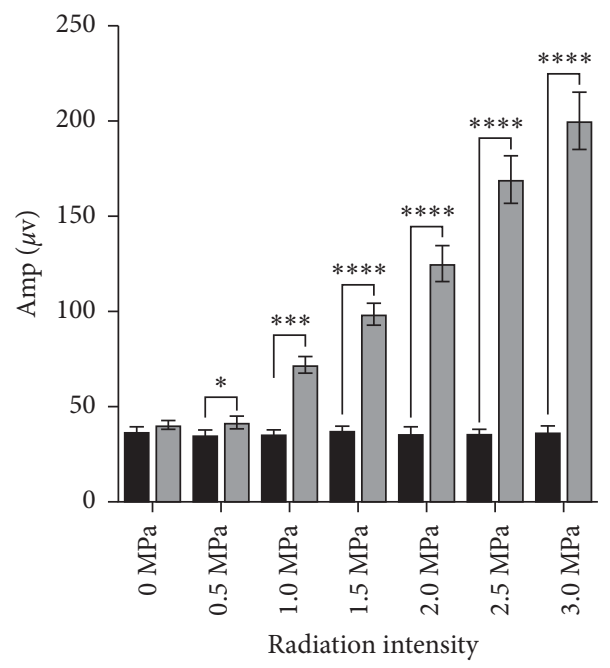

LIFU OFF

LIFU ON

(b)

FIGURE 3: Electromyography (EMG) shows the recruitment of the soleus (Sol) muscle by different intensities of LIFU stimulation. (a) The red arrow shows the moment at which the LIFU was initiated, and the black arrow shows the moment at which the LIFU was stopped. The duration (LIFU on) from the red arrow to the black arrow was $1 \mathrm{~s}$ and that from the black to the red arrow (LIFU turned off) was $4 \mathrm{~s}$. The black dotted rectangle shows the recruitment of Sol muscle and activation of EMG. (b) Amplitude of EMG after different irradiation intensity stimulations. ${ }^{*} P<0.05,{ }^{* * *} P<0.01$, and ${ }^{* * * *} P<0.0001$. Each symbol represents the mean \pm SEM; paired $t$-test; $n=6$ rats per assay.

3.2. LIFU Stimulation Enhances the Number of c-fos- and GAD65-Positive Cells. c-fos and GAD65 were used as markers of neuronal and synaptic activity, respectively. Neuronal activation can increase the number of c-fospositive cells and GAD65-positive cells [36, 37]. We found that the numbers of $c$-fos-positive cells increased after 0.5 $\mathrm{MPa}, 1.5 \mathrm{MPa}$, and 3.0 MPa stimulation and that those of GAD65-positive cells increased after $0.5 \mathrm{MPa}$ and $1.5 \mathrm{MPa}$ stimulation compared to stimulation with $0 \mathrm{MPa}$ (negative stimulation $)(P<0.05)$. However, the highest number of c-fos- and GAD65-positive cells was found in the $1.5 \mathrm{MPa}$ stimulation group (Figure 4).

3.3. Neuromotor Function and Electrophysiological Assessment. The safety of LIFU stimulation was examined at different irradiation intensities (0 MPa, 0.5 MPa, 1.5 MPa, and $3.0 \mathrm{MPa}$ ). After ultrasonic stimulation with different 


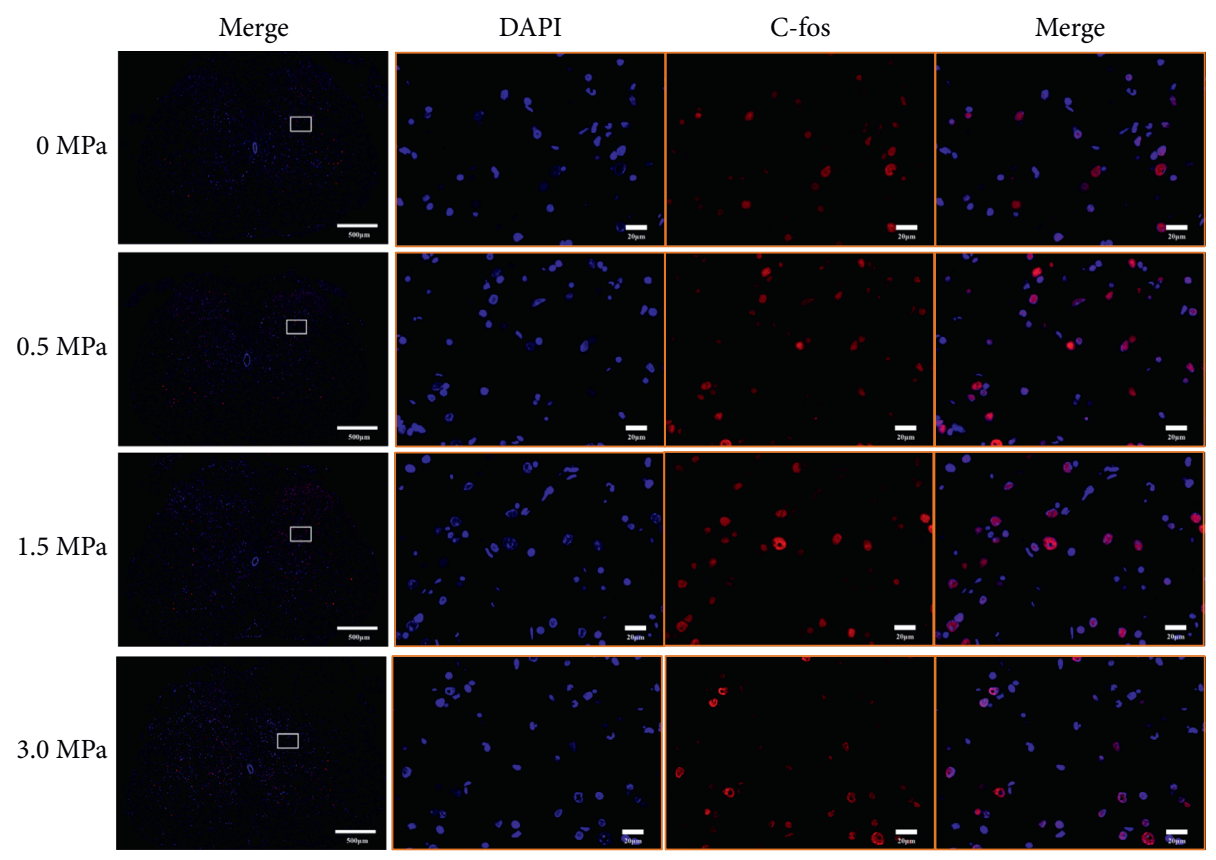

(a)

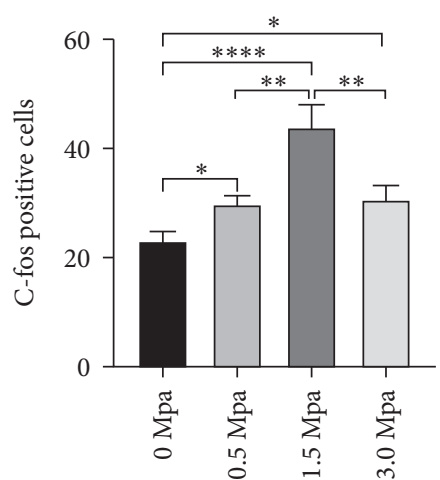

(b)

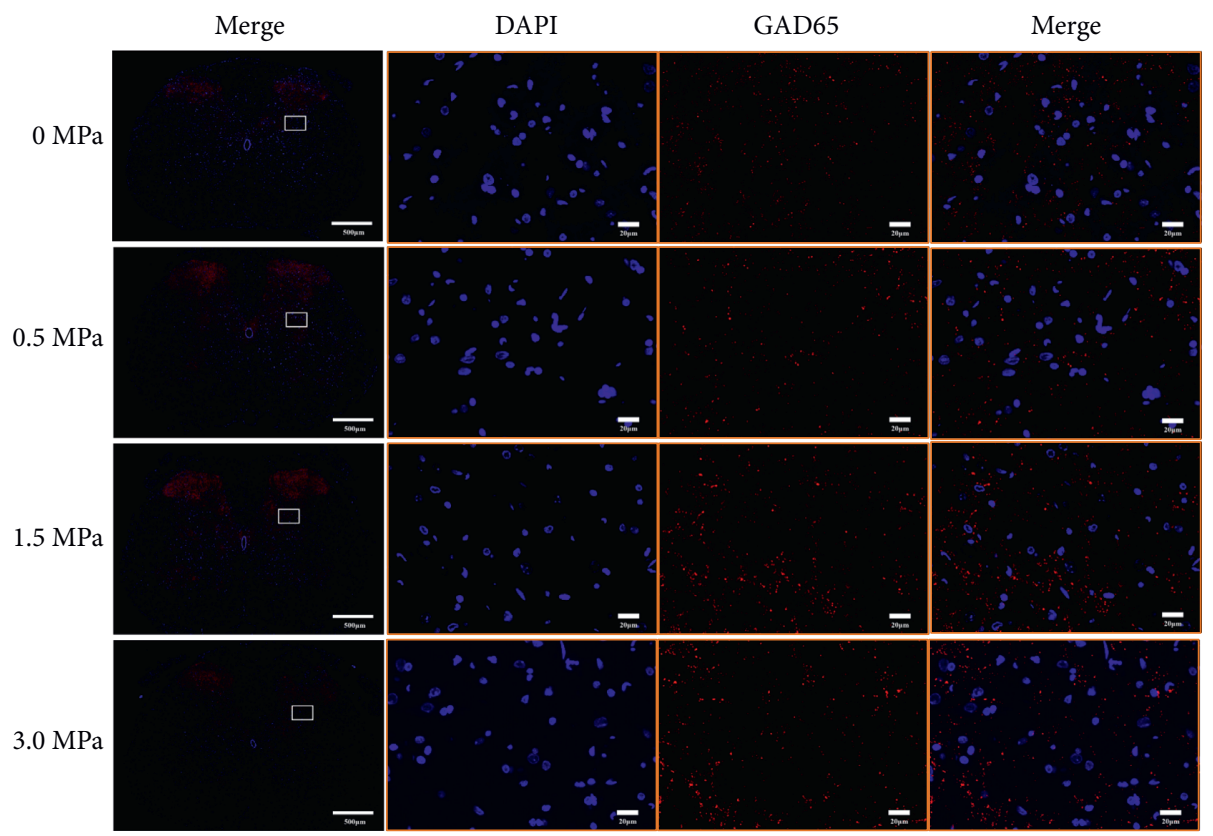

(c)

FIGURE 4: Continued. 


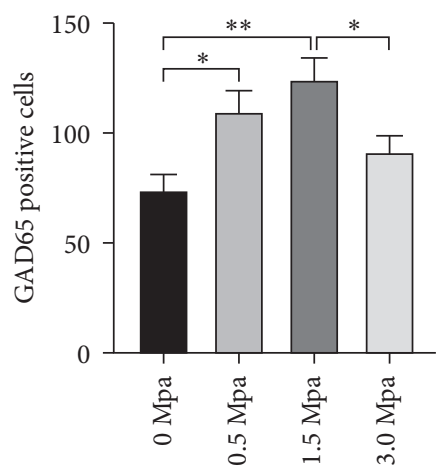

(d)

FIGURE 4: The effect of LIFU stimulation on neuron and synaptic activation in the lumbar spinal cord $(\times 40$ and $\times 400)$. Scale bar $=500 \mu \mathrm{m}$ and $20 \mu \mathrm{m}$. (a, c) Representative immunofluorescence pictures showing the c-fos-positive (a) and GAD65-positive (c) cells after different intensities of LIFU stimulation of the lumbar spinal cord. (b, d) C-fos- and GAD65-positive cells after different intensities of stimulation. ${ }^{*} P<0.05,{ }^{* * *} P<0.01$, and ${ }^{* * * *} P<0.0001$. Each symbol represents the mean \pm SEM; one-way ANOVA, followed by LSD test for pairwise comparisons; $n=3$ rats per assay.

intensities, the rats showed no changes in $\mathrm{BBB}$ score and latency of SEPs and MEPs (Supplementary Figure 2), except that the 3.0 MPa group had decreased SEP amplitudes (Figure 5).

\subsection{Inflammatory Factors and Histological Examination.} Spinal cord injuries can lead to high expression of inflammatory factors, such as IL- $1 \beta$ and TNF- $\alpha$. According to the ELISA results, stimulation with $0.5 \mathrm{MPa}$ and $1.5 \mathrm{MPa}$ LIFU did not significantly increase the expression of IL- $1 \beta$ and TNF- $\alpha$ compared to the negative stimulation $(0 \mathrm{MPa})$. In contrast, $3.0 \mathrm{MPa}$ stimulation significantly increased the expression of IL- $1 \beta$ and TNF- $\alpha$ compared to $0 \mathrm{MPa}$ and $0.5 \mathrm{MPa}$ irradiation intensities $(P<0.05)$ (Figure 6).

Among the LIFU $^{0+}$, LIFU ${ }^{1+}$, and LIFU ${ }^{2+}$ groups, we found no erythrocyte exudation (or bleeding), immune cell infiltration, or coagulative necrosis in $\mathrm{H}$ and $\mathrm{E}$ staining, no decrease in the numbers of Nissl bodies in Nissl staining, and no significant difference in the fluorescence intensity of GFAP (Figures 7-9). However, in the LIFU ${ }^{3+}$ group, H\&E staining revealed coagulation necrosis of the dorsal horn, especially at the right lateral portion (Figure 7). In the LIFU $^{3+}$ group, Nissl staining also showed significant necrosis on the right side of the dorsal horn, with structural destruction and loss of neurons, and the Nissl bodies showed condensation and darker staining (Figure 8). In the LIFU ${ }^{3+}$ group, the intensity of GFAP also increased compared to that of the $\mathrm{LIFU}^{0+}, \mathrm{LIFU}^{1+}$, and LIFU ${ }^{2+}$ groups $(P<0.05)$ (Figure 9).

3.5. Western Blot. We also examined the expression levels of caspase- 3 and $\mathrm{Bcl}-2$ as proapoptotic and antiapoptotic markers, respectively. According to the results of western blotting, the expression levels of caspase-3 and Bcl-2 remained unchanged on day 0 after different irradiation intensity stimulations $(P>0.05)$. On day 3 after LIFU stimulation, the $3.0 \mathrm{MPa}$ stimulation significantly increased the expression of caspase- 3 and Bcl-2 compared to $0 \mathrm{MPa}$ and $0.5 \mathrm{MPa}$ stimulations $(P<0.05)$, while there was no significant difference among $0 \mathrm{MPa}, 0.5 \mathrm{MPa}$, and $1.5 \mathrm{MPa}$ stimulations $(P>0.05)$ (Figure 10$)$.

\section{Discussion}

After Wall and Melzack [38] first proposed the concept that "control of pain may be achieved by selectively activating the large, rapidly conducting fibers," spinal cord stimulation has evolved significantly over the past decades [39]. Clinically, spinal cord stimulation or neuromodulation has attracted much attention in the management of chronic spinal conditions, especially for chronic spinal-related pain, such as failed back surgery syndrome/postlaminectomy syndrome, complex regional pain syndrome, and peripheral neuropathic pain [28]. In the past, spinal cord stimulation was induced by electrodes placed in the epidural space with pulse currents of different stimulation modalities, including highfrequency technology, bust stimulation, or other paradigms [40-43]. The electric field formed by electrical stimulation between the electrodes can transfer a specific amount of charge, thus altering the neuronal membrane potential, which is the basis of nerve recruitment [44]. The current study is the first to explore spinal cord neuromodulation with percutaneous LIFU stimulation.

The previous study found that LIFU stimulation of the brain or peripheral nerves can elicit electrophysiological changes [20]. For example, LIFU stimulation of the L5 dorsal root ganglion (DRG) or applied to a peripheral nerve in situ can also alter nerve function, including an increase in mechanical and thermal thresholds and suppression of compound action potentials and sensory action potentials in a neuropathic pain model $[45,46]$. Moreover, transcranial LIFU stimulation of the motor cortex activates neurons and evokes motor behavior, and the muscle contraction of limbs has been verified by EMG $[20,21]$. In this study, we successfully activated the neurocircuits of the spinal cord by 


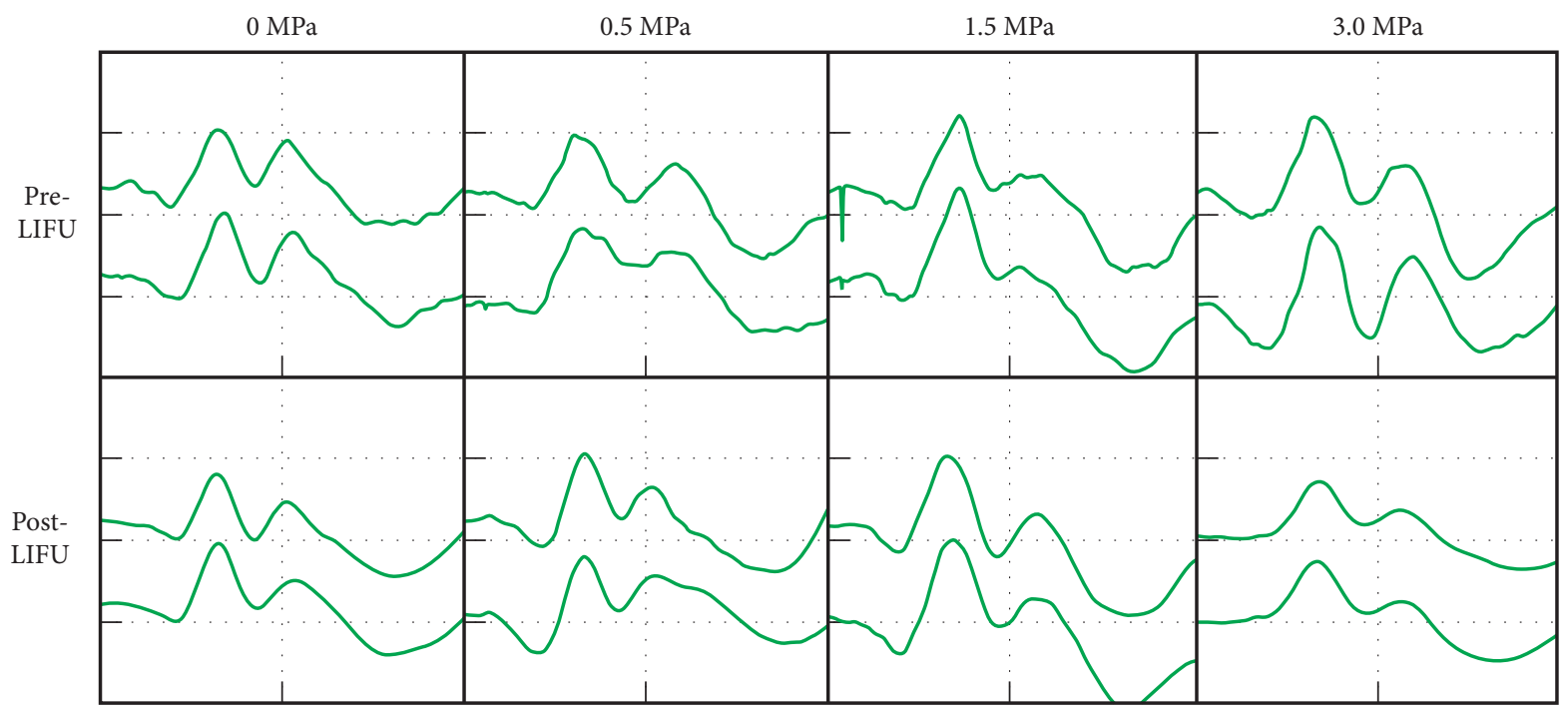

(a)

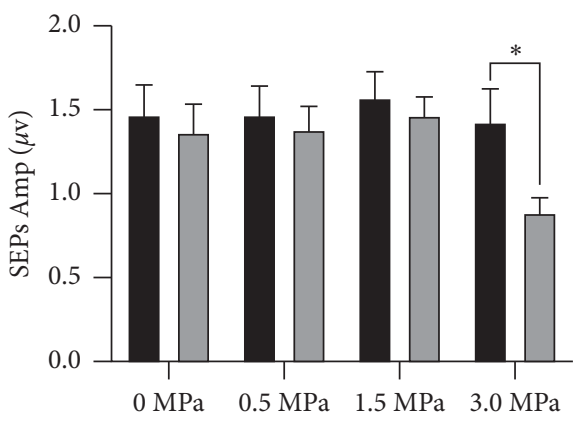

Pre-LIFU

$\square$ Post-LIFU

(b)

FIGURE 5: (a) Somatosensory evoked potentials (SEPs) were used to detect somatosensory conduction from the spinal cord. (b) SEP amplitude analyses for different intensities of stimulation. Each symbol represents the mean \pm SEM; paired $t$-test; $n=6$ rats per assay.

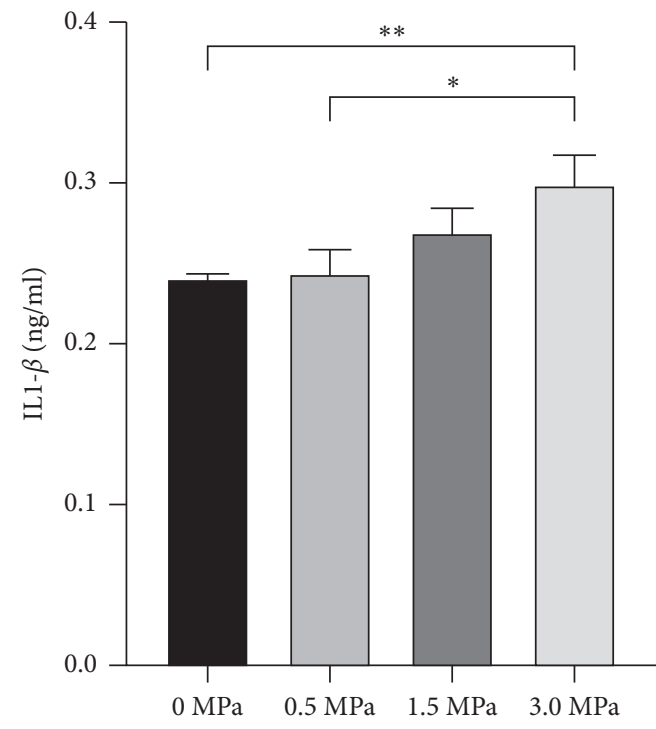

(a)

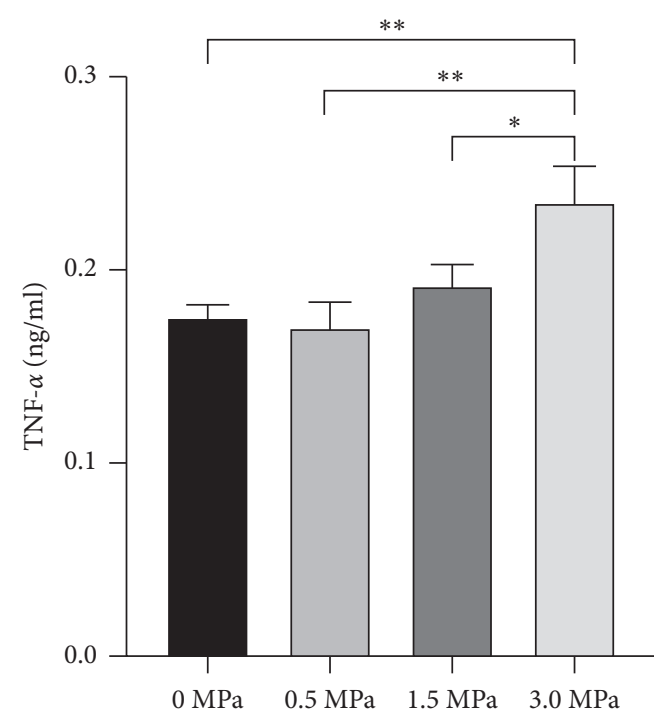

(b)

FIgURe 6: Enzyme-linked immunosorbent assay (ELISA) for detection of the inflammatory factors IL- $1 \beta$ (a) and TNF- $\alpha$ (b). ${ }^{*} P<0.05$ and ${ }^{* *} P<0.01$. Each symbol represents the mean \pm SEM; one-way ANOVA, followed by LSD test for pairwise comparisons; $n=3$ rats per assay. 


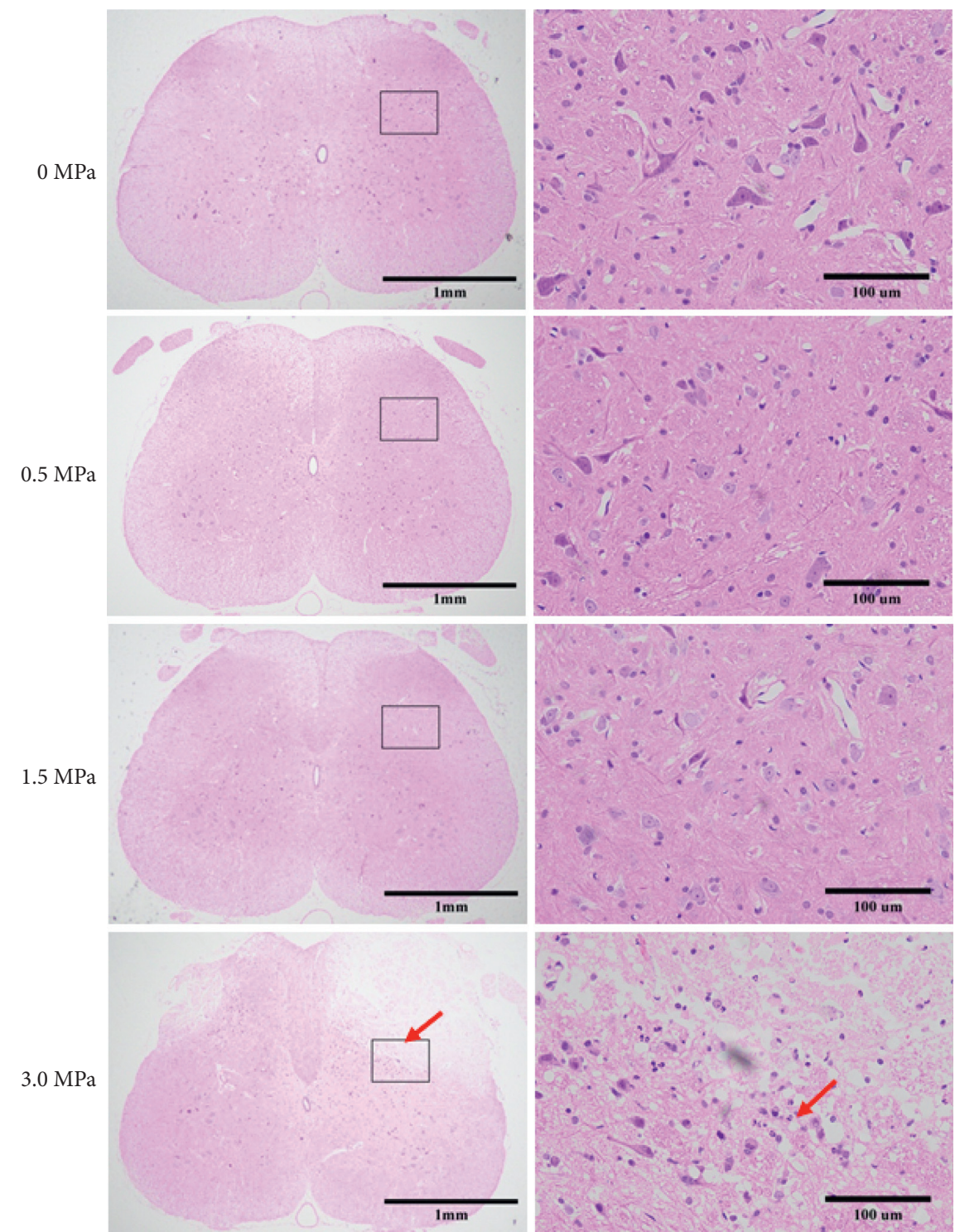

Figure 7: Hematoxylin and eosin (H\&E) staining for histological examination of spinal cord injury $(\times 40$ and $\times 400)$. Scale bars $=1 \mathrm{~mm}$ and $100 \mu \mathrm{m}$. Comparison of H\&E staining among the spinal cord sections after different irradiation intensities of LIFU stimulation. There was no significant difference in histological results among $0 \mathrm{MPa}, 0.5 \mathrm{MPa}$, and $1.5 \mathrm{MPa}$ stimulation groups. In the $3.0 \mathrm{MPa}$ stimulation group, coagulative necrosis was clear at the dorsal horn of the spinal cord (red arrow).

percutaneous stimulation with LIFU when the intensity was $>0.5 \mathrm{MPa}$. Spinal cord activation also induced the recruitment of Sol muscle as measured by EMG.

A previous study has shown that low-intensity ultrasound stimulation can also induce changes in biomarkers. For example, low-intensity ultrasound stimulation of peripheral nerves promoted injured nerve regeneration by stimulating the release of brain-derived neurotrophic factor (BDNF) [47]. Furthermore, transcranial focused ultrasound can decrease the expression of c-fos and increase the expression of GAD65 in the brains of SD rats with epilepsy, indicating that focused ultrasound deactivates excitatory cells and activates GABAergic terminals [37]. Another study has demonstrated that spinal cord activation increases the expression of c-fos and produces analgesia [36]. The increased numbers of c-fos- and GAD65-positive cells indicated the activation of neurons and synapses [36, 37]. In this study, we found that transdermal LIFU increased the numbers of c-fos- and GAD65-positive cells. From the results of EMG and biomarker studies (c-fos and GAD65), we hypothesize that the spinal cord was activated by LIFU.

Our results showed some differences from those of Chen, which showed that transcranial focused ultrasound reduced the number of c-fos-positive cells [37]; the difference may reflect the different animal models adopted. Tissue injury, such as paw inflammation [48], sciatic nerve transection [49], and chronic constriction injury of the sciatic nerve [50], can also increase c-fos expression. A previous study found that the expression of $\mathrm{c}$-fos increased in rats with seizures [51]. However, in this study, normal rats were used, and spinal cord activation by LIFU increased the number of c-fos-positive cells. 


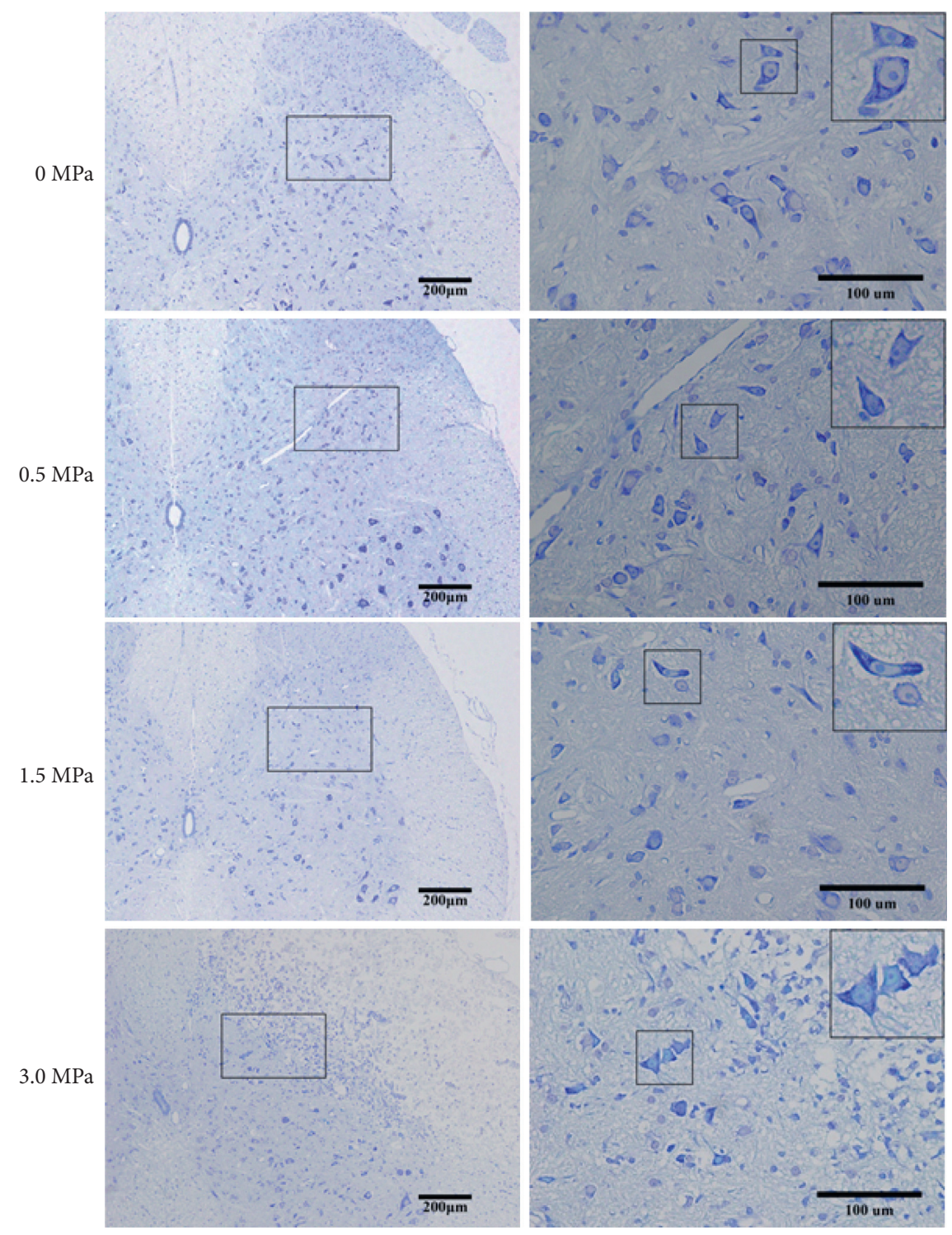

FIGURE 8: Nissl staining for histological examination of spinal cord injury $(\times 100$ and $\times 400)$. Scale bars $=200 \mu \mathrm{m}$ and $100 \mu \mathrm{m}$. Comparison of Nissl staining among the spinal cord sections after different irradiation intensities of LIFU stimulation. After $0 \mathrm{MPa}, 0.5 \mathrm{MPa}$, and $1.5 \mathrm{MPa}$ stimulation, the neuron arrangement was regular, the structure was clear, the morphology was normal, and the Nissl body was clear (shown in the black square). After 3.0 MPa irradiation intensity stimulation, the neuron arrangement was irregular, the structure was unclear, the morphology was abnormal, and the Nissl body was unclear (shown in the black square).

Previous studies have shown the many advantages of focused ultrasound stimulation, including high spatial resolution, noninvasive neurostimulation, and effective stimulation of the deep tissues with submillimeter static resolution [7, 8]. Moreover, it has been demonstrated that focused ultrasound can activate cells, brain histology slices, and neurocircuits of nonprimates, primates, and even humans $[13,16,19,20,22]$. However, the spinal cord is surrounded by irregular vertebral bones, which could lead to reflection, refraction, diffusion, and absorption of ultrasound beams, all of which may affect the neuromodulatory effects of ultrasound. In this study, we first demonstrated that LIFU enhances Sol muscle contractions and increases the number of c-fos- and GAD65-positive cells, suggesting activation of the spinal cord neurocircuits. The activation of the spinal cord neurocircuits by ultrasound may be due to the use of focused ultrasound, which can converge ultrasound beams onto the target to produce tissue effects [52]. Clearly, the activation of the spinal cord neurocircuits by low-intensity focused ultrasound would also provide an innovative and noninvasive neuromodulation method for spinal cord stimulation.

Safety is an important consideration in neuromodulation. Previous studies have demonstrated that ultrasound can induce different biological effects depending on the exposure parameters; for example, low-intensity ultrasound produces reversible cellular effects, whereas high-intensity ultrasound leads to irreversible cell death. According to the Food and Drug Administration (FDA), the $I_{\text {spta }}$ of the diagnostic ultrasound must be $\leq 720 \mathrm{mw} / \mathrm{cm}^{2}$ [53], 


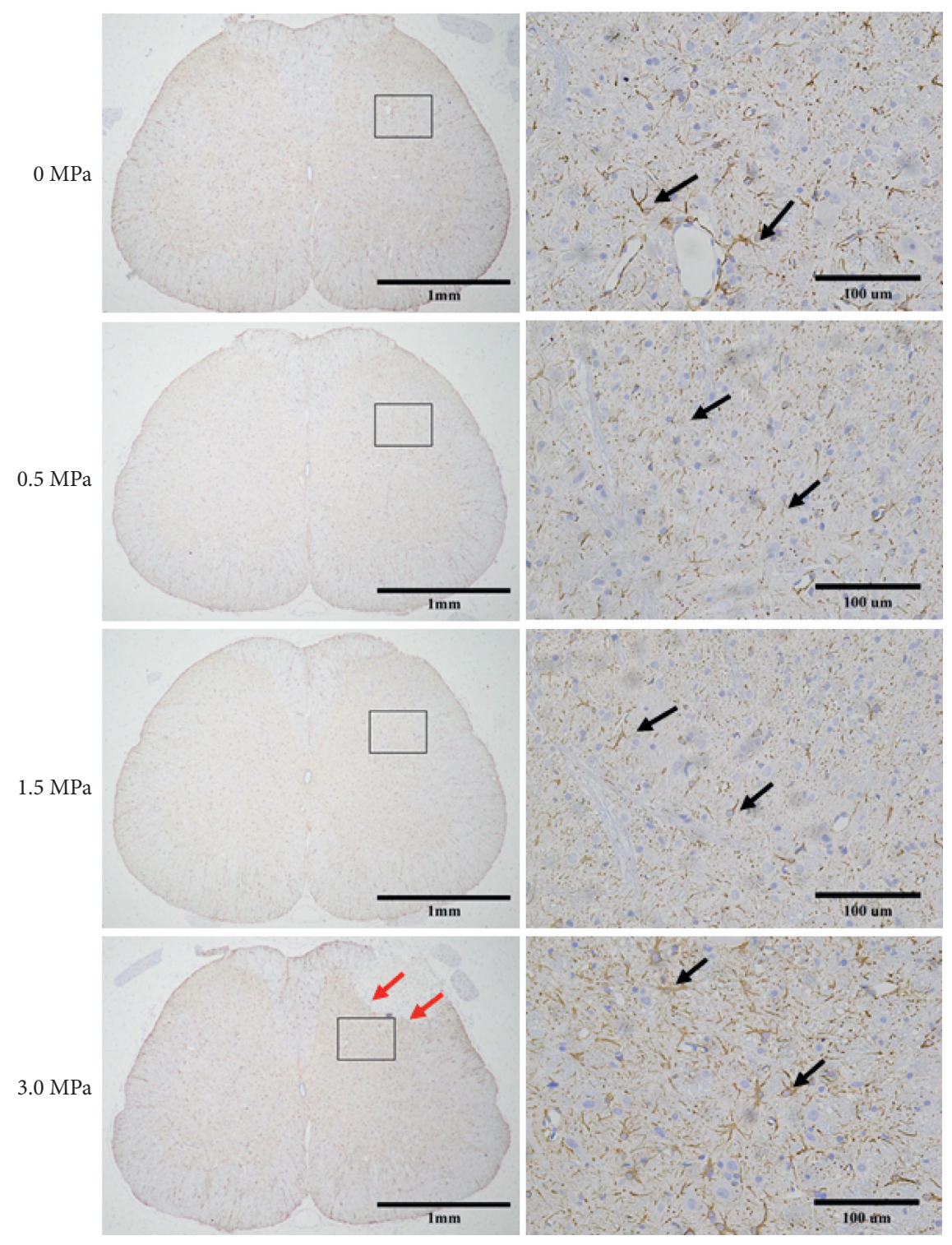

(a)

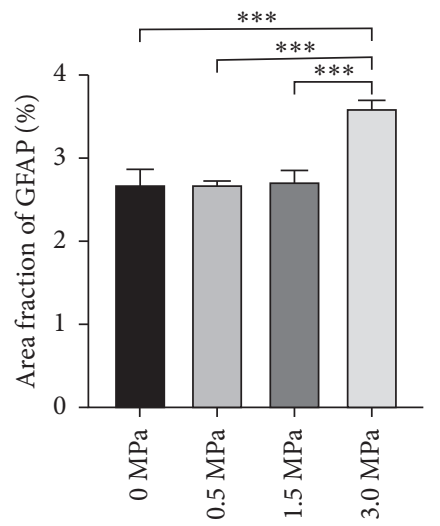

(b)

FIGURE 9: Results of immunohistochemistry (IHC) staining of astrocyte activation in the spinal cord sections after different irradiation intensities of LIFU stimulation $(\times 40$ and $\times 400)$. Scale bars $=1 \mathrm{~mm}$ and $100 \mu \mathrm{m}$. (a) Black arrows show the GFAP-positive astrocytes, and the red arrow shows coagulative necrosis of the spinal cord. A diagram indicating a $300 \mu \mathrm{m} \times 300 \mu \mathrm{m}$ square area was defined for further analysis of the positive cells. (b) Intensity analysis of the GFAP-positive area using ImageJ showed that 3.0 MPa stimulation increased the intensity of GFAP. ${ }^{* * *} P<0.0001$. Each symbol represents the mean \pm SEM; one-way ANOVA, followed by LSD test for pairwise comparisons; $n=3$ rats per assay.

which has been recognized as the requirement for ultrasonic neuromodulation treatment protocols. The safety of the FDA-recommended limit of ultrasound intensity has been demonstrated by previous studies. Some studies have shown that ultrasound intensity above the FDA limits but below the International Electrotechnical Commission (IEC) set intensity $\left(I_{\text {spta }}=3 \mathrm{~W} / \mathrm{cm}^{2}\right)$ for diagnostic medical ultrasound equipment is still safe [54]. Focused ultrasound with an intensity of $3.2 \mathrm{MPa}$ has also been used to stimulate the peripheral nervous system of the mice, activation of which was detected by EMG, while histological examination showed no evidence of nerve damage [55]. In this study, the neuromotor function, electrophysiology, H\&E staining, Nissl staining, and protein expression analyses suggested no injury to the spinal cord after $0.5 \mathrm{MPa}$ or $1.5 \mathrm{MPa}$ stimulation. Moreover, ultrasound has little thermal effect and does not cause tissue injury $[10,11]$. However, in this study, $3 \mathrm{MPa}$ stimulation decreased the SEP amplitude and increased the expression levels of IL- $1 \beta$, TNF- $\alpha$, caspase- 3 and Bcl-2, and GFAP, but it did not affect the neuromotor function or latency of SEPs and MEPs. The decreased amplitude of SEPs indicates damage to the spinal cord sensory pathways. The electrophysiological results were consistent with the H\&E staining results, which showed 


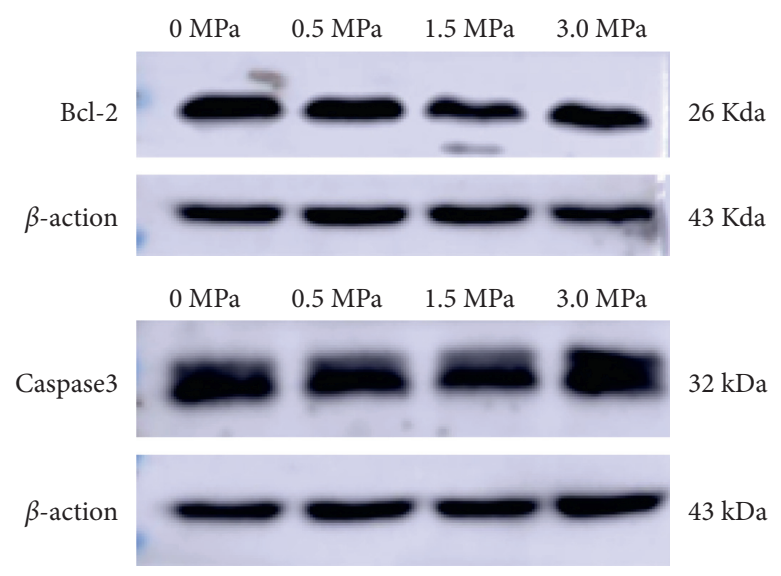

(a)

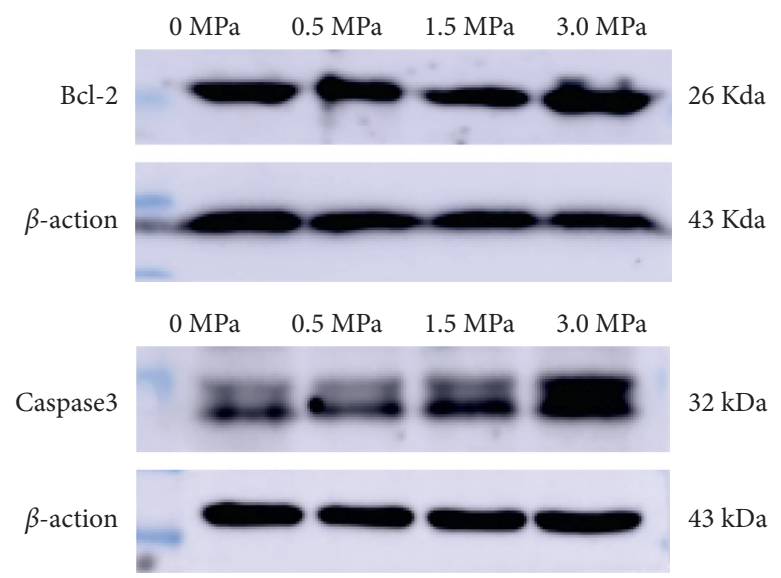

(d)
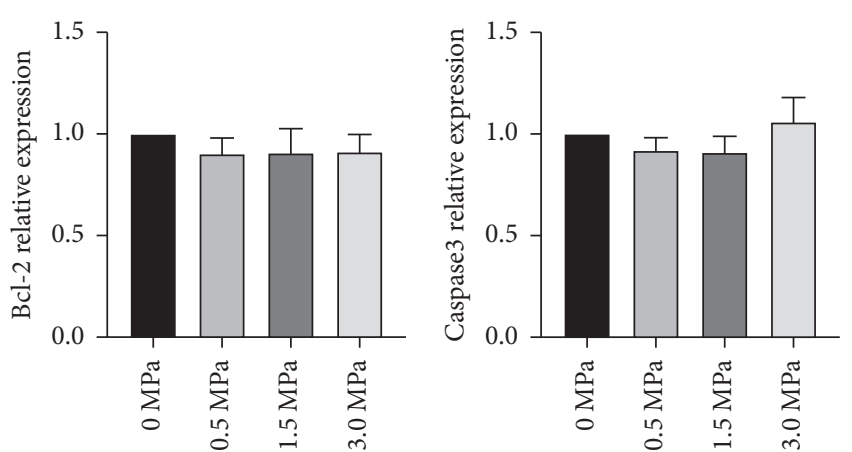

(b)

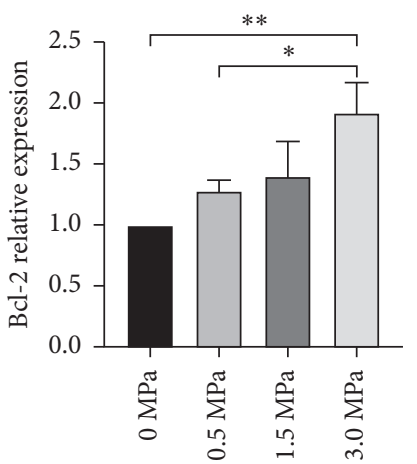

(e) (c)

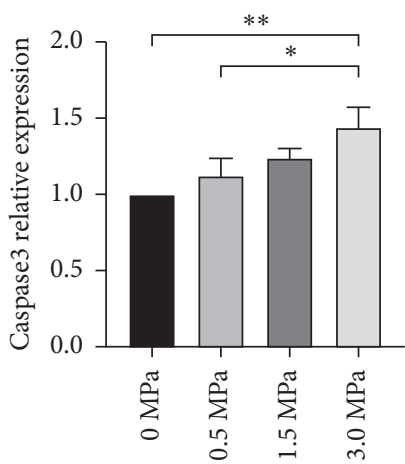

(f)

FIGURE 10: LIFU stimulation induces the expression of caspase- 3 and $\mathrm{Bcl}-2$ as markers of apoptosis induction and apoptosis inhibition. (a-c) Western blot results showed that LIFU stimulation did not significantly alter the expression levels of caspase-3 and Bcl-2 on day $0(P>0.05)$, while (d-f) $3.0 \mathrm{MPa}$ irradiation intensity stimulation increased the expression levels of caspase- 3 and $\mathrm{Bcl}-2$ compared to $0 \mathrm{MPa}$ and $0.5 \mathrm{MPa}$ irradiation intensities on day 3 after LIFU stimulation $(P<0.05)$. There were no significant differences among the $0 \mathrm{MPa}, 0.5 \mathrm{MPa}$, and 1.5 MPa irradiation intensity stimulation groups $(P>0.05)$. Values were normalized to $\beta$-actin. Each symbol represents the mean \pm SEM; ${ }^{*} P<0.05,{ }^{* *} P<0.01$; one-way ANOVA, followed by LSD test for pairwise comparisons; $n=3$ rats per assay.

coagulative necrosis of the dorsal horn. Furthermore, the WB results showed increased expression of caspase-3, confirming the apoptosis of neurons after spinal cord injury. The increased expression of $\mathrm{Bcl}-2$ suggests that the spinal cord produced an antioxidant response following injury. The differences observed between this study and previous studies may be due to various factors. First, focused ultrasound forms a focal spot in the deep tissue, which has a higher acoustic pressure and stimulation intensity and can produce stronger biological effects. Second, the spinal cord may exhibit a lower tolerance to ultrasonic stimulation than the peripheral nervous system. Finally, we suggest that the irradiation intensity should be strictly controlled when using low-intensity focused ultrasound to stimulate the central nervous system to avoid irreversible neural damage.

\section{Limitations}

This study has several limitations that warrant discussion. First, although we found that percutaneous low-intensity focused ultrasound activates spinal cord neurocircuits, the detailed mechanism is still unknown. Second, the prestudy confirmed that the temperature change was less than $0.025^{\circ} \mathrm{C}$ after LIFU stimulation. However, focused ultrasound can lead to spinal cord injury at an irradiation intensity of $3 \mathrm{MPa}$, and further experiments are needed to clarify the mechanisms underlying spinal cord injuries. Third, we confirmed that LIFU stimulation activates spinal cord neurocircuits, but we did not extend this research to determine how long the effects of LIFU stimulation are maintained. 


\section{Conclusions}

Noninvasive LIFU can effectively activate spinal cord neurocircuits and represents a safe neuromodulation method for spinal cord stimulation when the radiation intensity is $<1.5 \mathrm{MPa}$.

\section{Abbreviations}

LIFU: Low-intensity focused ultrasound

EMG: Electromyography

RI: Radiation intensity

BBB scale: Basso-Beattie-Bresnahan locomotor rating scale

Sol: $\quad$ Soleus

SEPs: $\quad$ Somatosensory evoked potentials

MEPs: $\quad$ Motor evoked potentials

Amp: Amplitude

H\&E: $\quad$ Hematoxylin and eosin.

\section{Data Availability}

The data used to support the findings of this study are included within the article.

\section{Conflicts of Interest}

The authors declare that they have no conflicts of interest.

\section{Acknowledgments}

This study was supported by the National Natural Science Foundation of China (nos. 81960421 and 82060421), the Science and Technology Innovative Team Grant of Kunming Medical University (no. CXTD201905), and Basic Research Program of Yunnan Science and Technology Department (no. 202101AT070255). The authors also thank LetPub (https://www.letpub.com) for the linguistic assistance during the preparation of this manuscript.

\section{Supplementary Materials}

Supplementary Figure 1: the amplitude of EMG after different irradiation intensity stimulations. ${ }^{*} P<0.05$ and ${ }^{* * * *} P<0.0001$. Each symbol represents the mean \pm SEM; one-way ANOVA, followed by LSD test for pairwise comparisons; $n=6$ rats per assay. Supplementary Figure 2: (a) the Basso, Beattie, and Bresnahan locomotor rating scale (BBB scale) was used to assess the neuromotor function of the hind limbs and the latency of (b) somatosensory evoked potentials (SEPs) and (d) motor evoked potentials (MEPs) evaluation for the spinal cord. The results show no difference in neuromotor function, latency of SEPs, and latency of MEPs after the different irradiation intensity stimulation $(P>0.05)$. (c) MEPs were used to detect motor conduction for the spinal cord, and the triangle represents the excitation point time. Each symbol represents the mean \pm SEM; paired $t$-test; $n=6$ rat per assay. (Supplementary Materials)

\section{References}

[1] P. B. Fitzgerald and Z. J. Daskalakis, "A practical guide to the use of repetitive transcranial magnetic stimulation in the treatment of depression," Brain Stimulation, vol. 5, no. 3, pp. 287-296, 2012.

[2] C. Ridler, "Spinal stimulation and physical therapy helps paraplegic patients to walk again," Nature Reviews Neurology, vol. 14, no. 11, p. 631, 2018.

[3] S. Li, M. Alam, R. U Ahmed et al., "Ultrasound-driven piezoelectric current activates spinal cord neurocircuits and restores locomotion in rats with spinal cord injury," Bioelectronic Medicine, vol. 6, no. 13, p. 13, 2020.

[4] P. Faria, M. Hallett, and P. C. Miranda, "A finite element analysis of the effect of electrode area and inter-electrode distance on the spatial distribution of the current density in tDCS," Journal of Neural Engineering, vol. 8, no. 6, Article ID 066017, 2011.

[5] F. Zhang, A. M. Aravanis, A. Adamantidis, L. De Lecea, and K. Deisseroth, "Circuit-breakers: optical technologies for probing neural signals and systems," Nature Reviews Neuroscience, vol. 8, no. 8, pp. 577-581, 2007.

[6] E. S. Boyden, "A history of optogenetics: the development of tools for controlling brain circuits with light," F1000 Biology Reports, vol. 3, p. 11, 2011.

[7] J. Mueller, W. Legon, A. Opitz, T. F. Sato, and W. J. Tyler, "Transcranial focused ultrasound modulates intrinsic and evoked EEG dynamics," Brain Stimulation, vol. 7, no. 6, pp. 900-908, 2014.

[8] S.-S. Yoo, A. Bystritsky, J.-H. Lee et al., "Focused ultrasound modulates region-specific brain activity," Neuroimage, vol. 56, no. 3, pp. 1267-1275, 2011.

[9] P. Bowary and B. D. Greenberg, "Noninvasive focused ultrasound for neuromodulation," Psychiatric Clinics of North America, vol. 41, no. 3, pp. 505-514, 2018.

[10] G. Ter Haar, "Therapeutic applications of ultrasound," Progress in Biophysics and Molecular Biology, vol. 93, no. 1-3, pp. 111-129, 2007.

[11] W. D. O’Brien, "Ultrasound-biophysics mechanisms," Progress in Biophysics and Molecular Biology, vol. 93, no. 1-3, pp. 212-255, 2007.

[12] W. J. Tyler, Y. Tufail, M. Finsterwald, M. L. Tauchmann, E. J. Olson, and C. Majestic, "Remote excitation of neuronal circuits using low-intensity, low-frequency ultrasound," PLoS One, vol. 3, no. 10, Article ID e3511, 2008.

[13] Z. Lin, X. Huang, W. Zhou et al., "Ultrasound stimulation modulates voltage-gated potassium currents associated with action potential shape in hippocampal CA1 pyramidal neurons," Frontiers in Pharmacology, vol. 10, p. 544, 2019.

[14] J. Vion-Bailly, W. A. N'Djin, I. M. Suarez Castellanos, J.-L. Mestas, A. Carpentier, and J.-Y. Chapelon, "A causal study of the phenomenon of ultrasound neurostimulation applied to an in vivo invertebrate nervous model," Scientific Reports, vol. 9, no. 1, p. 13738, 2019.

[15] W. Zhou, J. Wang, K. Wang et al., "Ultrasound neuromodulation chip: activation of sensory neurons in Caenorhabditis elegans by surface acoustic waves," Lab on a Chip, vol. 17, no. 10, pp. 1725-1731, 2017.

[16] W. Legon, T. F. Sato, A. Opitz et al., "Transcranial focused ultrasound modulates the activity of primary somatosensory cortex in humans," Nature Neuroscience, vol. 17, no. 2, pp. 322-329, 2014.

[17] G. Leinenga, C. Langton, R. Nisbet, and J. Götz, "Ultrasound treatment of neurological diseases-current and emerging 
applications," Nature Reviews Neurology, vol. 12, no. 3, pp. 161-174, 2016.

[18] R. F. Dallapiazza, K. F. Timbie, Holmberg et al., "Noninvasive neuromodulation and thalamic mapping with low-intensity focused ultrasound," Journal of Neurosurgery, vol. 128, no. 3, pp. 875-884, 2018.

[19] W. Lee, S. D. Lee, Park et al., "Image-guided focused ultrasound-mediated regional brain stimulation in sheep," $U l$ trasound in Medicine \& Biology, vol. 42, no. 2, pp. 459-470, 2016.

[20] R. L. King, J. R. Brown, W. T. Newsome, and K. B. Pauly, "Effective parameters for ultrasound-induced in vivo neurostimulation," Ultrasound in Medicine \& Biology, vol. 39, no. 2, pp. 312-331, 2013.

[21] Y. Tufail, A. Matyushov, Baldwin et al., "Transcranial pulsed ultrasound stimulates intact brain circuits," Neuron, vol. 66, no. 5, pp. 681-694, 2010.

[22] T. Deffieux, Y. Younan, N. Wattiez, M. Tanter, P. Pouget, and J.-F. Aubry, "Low-intensity focused ultrasound modulates monkey visuomotor behavior," Current Biology, vol. 23, no. 23, pp. 2430-2433, 2013.

[23] L. R. Gavrilov, E. M. Tsirulnikov, and I. A. I. Davies, "Application of focused ultrasound for the stimulation of neural structures," Ultrasound in Medicine \& Biology, vol. 22, no. 2, pp. 179-192, 1996.

[24] K. Meier, "Spinal cord stimulation: background and clinical application," Scandinavian Journal of Pain, vol. 5, no. 3, pp. 175-181, 2014.

[25] K. Chakravarthy, H. Richter, P. J. Christo, K. Williams, and Y. Guan, "Spinal cord stimulation for treating chronic pain: reviewing preclinical and clinical data on paresthesia-free high-frequency therapy," Neuromodulation: Technology at the Neural Interface, vol. 21, no. 1, pp. 10-18, 2018.

[26] A. D. Sdrulla, Y. Guan, and S. N. Raja, "Spinal cord stimulation: clinical efficacy and potential mechanisms," Pain Practice, vol. 18, no. 8, pp. 1048-1067, 2018.

[27] C. Willyard, "How a revolutionary technique got people with spinal-cord injuries back on their feet," Nature, vol. 572, no. 7767 , pp. 20-25, 2019.

[28] G. L. Schmidt, "The use of spinal cord stimulation/neuromodulation in the management of chronic pain," Journal of the American Academy of Orthopaedic Surgeons, vol. 27, no. 9, pp. e401-e407, 2019.

[29] Y. H. Liao, B. Wang, M. X. Chen, Y. Liu, and L. J. Ao, "LIFU alleviates neuropathic pain by improving the KCC2 expression and inhibiting the CaMKIV-KCC2 pathway in the L4-L5 section of the spinal cord," Neural Plast, vol. 2021, Article ID 6659668, 10 pages, 2021.

[30] D. M. Basso, M. S. Beattie, and J. C. Bresnahan, "A sensitive and reliable locomotor rating scale for open field testing in rats," Journal of Neurotrauma, vol. 12, no. 1, pp. 1-21, 1995.

[31] J. A. Corleto, M. Bravo-Hernández, Kamizato et al., “Thoracic 9 spinal transection-induced model of muscle spasticity in the rat: a systematic electrophysiological and histopathological characterization," PLoS One, vol. 10, no. 12, Article ID e0144642, 2015.

[32] H. Beverungen, S. C. Klaszky, M. Klaszky, and M.-P. Côté, "Rehabilitation decreases spasticity by restoring chloride homeostasis through the brain-derived neurotrophic factorKCC2 pathway after spinal cord injury," Journal of Neurotrauma, vol. 37, no. 6, pp. 846-859, 2020.

[33] T.-Y. Chen, S.-H. Tai, E.-J. Lee et al., "Cinnamophilin offers prolonged neuroprotection against gray and white matter damage and improves functional and electrophysiological outcomes after transient focal cerebral ischemia*," Critical Care Medicine, vol. 39, no. 5, pp. 1130-1137, 2011.

[34] M. G. Schlag, R. Hopf, and H. Redl, "Serial recording of sensory, corticomotor, and brainstem-derived motor evoked potentials in the rat," Somatosensory \& Motor Research, vol. 18, no. 2, pp. 106-116, 2001.

[35] Y. Liu, G. Lu, Su et al., "Characterization of axon damage, neurological deficits, and histopathology in two experimental models of intracerebral hemorrhage," Frontiers in Neuroscience, vol. 12, p. 928, 2018.

[36] R. Melzack and P. D. Wall, "Pain mechanisms: a new theory," Science, vol. 150, no. 3699, p. 971, 1965.

[37] T. R. Deer, E. Krames, N. Mekhail et al., "The appropriate use of neurostimulation: new and evolving neurostimulation therapies and applicable treatment for chronic pain and selected disease states," Neuromodulation: Technology at the Neural Interface, vol. 17, no. 6, pp. 599-615, 2014.

[38] L. Kapural, C. Yu, M. W. Doust et al., "Comparison of 10-kHz high-frequency and traditional low-frequency spinal cord stimulation for the treatment of chronic back and leg pain," Neurosurgery, vol. 79, no. 5, pp. 667-677, 2016.

[39] S. Hou, K. Kemp, and M. Grabois, "A systematic evaluation of burst spinal cord stimulation for chronic back and limb pain," Neuromodulation: Technology at the Neural Interface, vol. 19, no. 4, pp. 398-405, 2016.

[40] E. Veizi, S. M. Hayek, J. North et al., "Spinal cord stimulation (SCS) with anatomically guided (3D) neural targeting shows superior chronic axial low back pain relief compared to traditional SCS-LUMINA study," Pain Medicine (Malden, Mass), vol. 18, no. 8, pp. 1534-1548, 2017.

[41] J. P. Miller, S. Eldabe, E. Buchser, L. M. Johanek, Y. Guan, and B. Linderoth, "Parameters of spinal cord stimulation and their role in electrical charge delivery: a review," Neuromodulation: Technology at the Neural Interface, vol. 19, no. 4, pp. 373-384, 2016.

[42] R. Vallejo, k. Bradley, and L. Kapural, "Spinal cord stimulation in chronic pain: mode of action," Spine (Phila Pa 1976), vol. 42, no. 14, pp. s53-s60, 2017.

[43] Y.-F. Lee, C.-C. Lin, J.-S. Cheng, and G.-S. Chen, "High-intensity focused ultrasound attenuates neural responses of sciatic nerves isolated from normal or neuropathic rats," Ultrasound in Medicine \& Biology, vol. 41, no. 1, pp. 132-142, 2015.

[44] Y. Youn, A. Hellman, Walling et al., "High-intensity ultrasound treatment for vincristine-induced neuropathic pain," Neurosurgery, vol. 83, no. 5, pp. 1068-1075, 2018.

[45] X.-J. Ni, X.-D. Wang, Y.-H. Zhao et al., "The effect of lowintensity ultrasound on brain-derived neurotropic factor expression in a rat sciatic nerve crushed injury model," $U l-$ trasound in Medicine \& Biology, vol. 43, no. 2, pp. 461-468, 2017.

[46] S.-G. Chen, C.-H. Tsai, C.-J. Lin et al., "Transcranial focused ultrasound pulsation suppresses pentylenetetrazol induced epilepsy in vivo," Brain Stimulation, vol. 13, no. 1, pp. 35-46, 2020.

[47] Y. Maeda, M. Ikeuchi, P. Wacnik, and K. A. Sluka, "Increased c-fos immunoreactivity in the spinal cord and brain following spinal cord stimulation is frequency-dependent," Brain Research, vol. 1259, pp. 40-50, 2009.

[48] M. Pinto, D. Lima, and I. Tavares, "Neuronal activation at the spinal cord and medullary pain control centers after joint stimulation: a c-fos study in acute and chronic articular inflammation," Neuroscience, vol. 147, no. 4, pp. 1076-1089, 2007. 
[49] S.-I. Chi, J. D. Levine, and A. I. Basbaum, "Peripheral and central contributions to the persistent expression of spinal cord fos-like immunoreactivity produced by sciatic nerve transection in the rat," Brain Research, vol. 617, no. 2, pp. 225-237, 1993.

[50] K. C. Kajander, A. M. Madsen, M. J. Iadarola, G. Draisci, and S. Wakisaka, "Fos-like immunoreactivity increases in the lumbar spinal cord following a chronic constriction injury to the sciatic nerve of rat," Neuroscience Letters, vol. 206, no. 1, pp. 9-12, 1996.

[51] H. Yang, W. Shan, F. Zhu et al., "C-Fos mapping and EEG characteristics of multiple mice brain regions in pentylenetetrazol-induced seizure mice model," Neurological Research, vol. 41, no. 8, pp. 749-761, 2019.

[52] G. R. Giammalva, C. Gagliardo, S. Marrone et al., "Focused ultrasound in neuroscience. State of the art and future perspectives," Brain Science, vol. 11, no. 1, 2021.

[53] Administration USFaD, Marketing Clearance of Diagnostic Ultrasound Systems and Transducers. Draft Guidance for Industry and Food and Drug Administration Staff, Administration USFaD, Silver Spring, USA, 2017.

[54] F. A. Duck, "Medical and non-medical protection standards for ultrasound and infrasound," Progress in Biophysics and Molecular Biology, vol. 93, no. 1-3, pp. 176-191, 2007.

[55] M. E. Downs, S. A. Lee, G. Yang, S. Kim, Q. Wang, and E. E. Konofagou, "Non-invasive peripheral nerve stimulation via focused ultrasound in vivo," Physics in Medicine and Biology, vol. 63, no. 3, Article ID 035011, 2018. 